\title{
A Remark on an Infinite Tensor Product of von Neumann Algebras
}

\author{
By
}

Huzihiro Araki and Yoshiomi NaKagami*

\begin{abstract}
Let $H_{\mathfrak{c}}$ be the incomplete infinite tensor product of Hilbert spaces $H_{\text {c con- }}$ taining a product vector $\otimes x_{\iota}$, where $c$ denotes the equivalence class of the $\mathfrak{夭}_{0}$-sequence $\left\{x_{\imath}\right\}$. Let $E_{\mathfrak{F}}$ be the projection on $H_{\mathfrak{F}}$ in the complete infinite tensor product $H$ of $H_{\iota}$. Let $\Re$ be the von Neumann algebra on $H$ generated by von Neumann algebra $\mathfrak{R}$, on $H_{\text {c }}$ and $E(\mathfrak{c})$ be the central support of $E_{\mathrm{q}}$ in $\mathfrak{R}^{\prime}$. Two $\mathfrak{E}_{0}$-sequences $\left\{x_{\iota}\right\}$ and $\left\{y_{\iota}\right\}$, and their equivalence classes $\mathfrak{c}$ and $\mathfrak{c}^{\prime}$, are defined to be $p$-equivalent if there exist partial isometries $p_{\imath} \in \mathfrak{R}_{\imath}^{\prime}$ such that $\left\{x_{\imath}\right\}$ and $\left\{p_{\iota} y_{\iota}\right\}$ are equivalent and $p_{\iota}^{*} p_{\iota} y_{\iota}=y_{\iota}$. They are defined to be $u$-equivalent if $p_{c}$ can be chosen unitary. We prove that $E(\mathrm{c})$ is the sum of $E_{\mathfrak{c}}$, with $c^{\prime}, p^{-}$ equivalent to $c$. If the index set is countable, $p$-equivalence and $u$-equivalence coincide.
\end{abstract}

\section{$\S 1$. Introduction}

According to von Neumann [8], the complete infinite tensor product $H=\otimes H_{\iota}$ of Hilbert spaces $H_{\iota}, \iota \in I$, is the (linear topological) span of all product vectors $\otimes x_{\iota}$ (multilinear in $x_{\iota}$ ) such that $x_{\iota} \neq 0$ and

$$
\sum\left|1-\left\|x_{\imath}\right\|\right|<\infty
$$

(We have substituted "tensor" into von Neumann's "direct".) Let $S$ denote the set of all $\left\{x_{\iota}\right\}$ satisfying (1.1) and $S_{0}$ denote the set of all $\left\{x_{\iota}\right\} \in S$ such that $x_{\iota} \neq 0 . \quad\left\{x_{\iota}\right\}$ and $\left\{y_{\iota}\right\}$ are called (strongly) equivalent if

Received June 20, 1972.

* Department of Mathematics, Tokyo Institute of Technology, Tokyo, Japan. 


$$
\sum\left|1-\left(x_{\iota}, y_{\iota}\right)\right|<\infty .
$$

Notation: $\left\{x_{\iota}\right\} \sim\left\{y_{\imath}\right\}$. It defines equivalence relations in $S$ and $S_{0}$. Let $\sqrt{5}$ and $\mathfrak{F}_{0}$ denote the set of equivalence classes $c\left(\left\{x_{\iota}\right\}\right)$ of $\left\{x_{\iota}\right\}$ in $S$ and $S_{0}$, respectively. The subspace of $H$ spanned by $\otimes x_{\iota}$ with a fixed $c\left(\left\{x_{\iota}\right\}\right)$ $=c \in \mathfrak{C}_{0}$ is called the incomplete infinite tensor product and is denoted by $H_{\mathrm{c}}=\otimes^{\mathrm{c}} H_{\iota}$. Let $E_{\mathrm{c}}$ denote the projection on $H_{\mathrm{c}}$ in $H$.

Let $\Re$ be a von Neumann algebra on $H_{\iota}, \pi$ be its natural representation on $H$ (namely $\pi(Q)\left(\otimes x_{\iota}\right)=\otimes x_{\iota}^{\prime}$, with $x_{\iota}^{\prime}=x_{\iota}$ for $\iota \neq \iota_{0}$ and $x_{\iota}^{\prime}=$ $Q x_{\iota}$ for $\iota=\iota_{0}$, if $\left.Q \in \Re_{\iota_{0}}\right)$. Let $\Re \equiv \bigotimes \Re$ be the von Neumann algebra generated by the union of all $\pi\left(\Re_{\iota}\right)$. Since $H_{c}$ is invariant under each $\pi\left(\Re_{\iota}\right), E_{c}$ is in $\Re^{\prime}$. Let $E(\mathfrak{c})$ be the central support of $E_{\mathrm{c}}$ in $\Re^{\prime}$.

Definition. Let $\left\{x_{\iota}\right\},\left\{y_{\imath}\right\} \in S$ and $\mathfrak{c}=\mathfrak{c}\left(\left\{x_{\iota}\right\}\right), \mathfrak{c}^{\prime}=\mathfrak{c}\left(\left\{y_{\iota}\right\}\right)$.

(1) $\left\{x_{\iota}\right\}$ and $c$ are u-equivalent to $\left\{y_{\iota}\right\}$ and $c^{\prime}$, respectively if $\left\{x_{\iota}\right\}$ $\sim\left\{u_{\iota} y_{\iota}\right\}$ for some unitary $u_{\iota} \in \Re_{\iota}^{\prime}$. Notation: $\left\{x_{\iota}\right\} \underset{u}{\sim}\left\{y_{\iota}\right\}, \mathfrak{c} \sim_{u} \mathfrak{c}^{\prime}$.

(2) $\left\{x_{\iota}\right\}$ and $c$ are p-equivalent to $\left\{y_{\iota}\right\}$ and $c^{\prime}$, respectively, if $\left\{x_{\iota}\right\}$ $\sim\left\{p_{\iota} y_{\iota}\right\}$ for some partial isometry $p_{\iota} \in \Re_{\iota}^{\prime}$ such that $p_{\iota}^{*} p_{\iota} y_{\iota}=y_{\iota}$. Notation: $\left\{x_{\iota}\right\} \underset{p}{\sim}\left\{y_{\imath}\right\}, \underset{p}{\mathfrak{c}} \mathcal{c}^{\prime}$.

(3) $\left\{x_{\iota}\right\}$ and $c$ are v-equivalent to $\left\{y_{\iota}\right\}$ and $c^{\prime}$, respectively, if $\left\{x_{\iota}\right\}$ $\sim\left\{v_{\iota} y_{\iota}\right\}$ for some $v_{\iota} \in \Re_{\iota}^{\prime}$ such that $\left\|v_{\iota}\right\|_{i} \leqq 1$. Notation: $\left\{x_{\iota}\right\} \underset{v}{\sim}\{y\}, \mathfrak{c} \underset{v}{\sim} \mathcal{c}^{\prime}$.

Our main result is the following:

Theorem. (1) $E(\mathrm{c})$ is the sum of $E_{c^{\prime}}$ with $\mathfrak{c}_{p}^{\prime} \sim$.

(2) If the index set I is countable, $\mathfrak{c}_{p}^{\prime} \underset{c}{\mathcal{c}}$ and $\mathfrak{c}^{\prime} \underset{u}{\mathcal{c}}$ are equivalent.

Remark. If $\Re_{\iota}=\mathscr{B}\left(H_{\iota}\right)$, the set of all bounded linear operators on $H_{\iota}$, then $\underset{u}{\sim}, \underset{p}{\sim}$ and $\underset{v}{\sim}$ all coincide with the weak equivalence introduced by von Neumann.

\section{§ 2. Equivalence Relations}

The $u$-equivalence is clearly an equivalence relation. In this section, we shall show that $p$ - and $v$-equivalence are also equivalence relations and 
are the same. In the definition of $v$-equivalence, we have not stated the condition $\left\{v_{\imath} y_{\imath}\right\} \in S$. This is actually a consequence of $\left\{x_{\imath}\right\} \in S,\left\{y_{\imath}\right\} \in S$ and $\left\{x_{\imath}\right\} \sim\left\{v_{\imath} y_{\imath}\right\}$, as is shown in the next Lemma.

Lemma 1. If $\left\{x_{\iota}\right\} \in S,\left\{y_{\iota}\right\} \in S,\left\|v_{\imath}\right\| \leqq 1$ and $\left\{x_{\iota}\right\} \sim\left\{v_{\iota} y_{\iota}\right\}$, then $\left\{v_{\iota} y_{\imath}\right\} \in S$.

Proof. Since $\left\{y_{\iota}\right\} \in S$ and $\left\|v_{\imath}\right\| \leqq 1$

$$
\sup \left\|v_{\imath} y_{\imath}\right\| \leqq \sup \left\|y_{\imath}\right\|<\infty
$$

If $\left\|v_{\imath} y_{\iota}\right\| \geqq 1$, then $0 \geqq 1-\left\|v_{\imath} y_{\iota}\right\| \geqq 1-\left\|y_{\imath}\right\|$ and hence

$$
1-\left\|v_{\imath} y_{\imath}\right\| \geqq\left|1-\left\|v_{\imath} y_{\imath}\right\|\right|-2\left|1-\left\|y_{\imath}\right\|\right| \text {. }
$$

This inequality obviously holds for $1 \geqq\left\|_{\imath} v_{\imath} y_{\imath}\right\|$. Now assume that $\left\{v_{\imath} y_{\iota}\right\}$ $\notin S$. Then

$$
\begin{aligned}
& \sum\left|1-\left(x_{\imath}, v_{\imath} y_{\iota}\right)\right| \geqq \sum\left\{1-\left|\left(x_{\iota}, v_{\imath} y_{\iota}\right)\right|\right\} \\
& \geqq \sum\left\{1-\left\|x_{\iota}\right\|\left\|v_{\iota} y_{\iota}\right\|\right\}=\sum\left(1-\left\|v_{\iota} y_{\iota}\right\|\right)+\sum\left\|v_{\iota} y_{\iota}\right\|\left(1-\left\|x_{\iota}\right\|\right) \\
& \geqq \sum\left|1-\left\|v_{\imath} y_{\imath}\right\|\right|-2 \sum\left|1-\left\|y_{\imath}\right\|\right|--\sup \left\|v_{\iota} y_{\imath}\right\| \sum\left|1-\left\|x_{\imath}\right\|\right| \\
& =+\infty
\end{aligned}
$$

which contradicts with $\left\{x_{\iota}\right\} \sim\left\{v_{\iota} y_{\iota}\right\}$.

Q.E.D.

Lemma 2. $\left\{x_{\imath}\right\} \underset{p}{\sim}\left\{y_{\imath}\right\}$ and $\left\{x_{\iota}\right\} \underset{v}{\sim}\left\{y_{\imath}\right\}$ are equivalent.

Proof. Obviously $\left\{x_{\imath}\right\} \underset{p}{\sim}\left\{y_{\iota}\right\}$ implies $\left\{x_{\imath}\right\} \underset{v}{\sim}\left\{y_{\imath}\right\}$. To prove the converse, let $\left\{x_{\iota}\right\} \sim\left\{v_{\iota} y_{\iota}\right\}$ with $\left\|v_{\imath}\right\| \leqq 1$. Let $s^{\prime}\left(y_{\iota}\right)$ denote the smallest projection $E=s^{\prime}\left(y_{\iota}\right) \in \Re_{\iota}^{\prime}$ such that $E y_{\iota}=y_{\iota}\left(E H_{\iota}\right.$ is the closure of $R_{\iota} y_{\iota}$. $)$ Let $p_{\iota} q_{\iota}=v_{\iota} s^{\prime}\left(y_{\iota}\right)$ be the polar decomposition with $q_{\iota}=\left|v_{\iota} s^{\prime}\left(y_{\iota}\right)\right|, p_{\iota}^{*} p_{\iota}=$ $s^{\prime}\left(q_{\iota}\right)$ (1 minus the spectral projection of $q_{\iota}$ for the eigenvalue 0 ).

Since $\left\|q_{\imath} y_{\imath}\right\|=\left\|v_{\imath} s^{\prime}\left(y_{\imath}\right) y_{\imath}\right\|=\left\|v_{\imath} y_{\imath}\right\|$ and $\left\{v_{\imath} y_{\imath}\right\} \in S$ by Lemma 1 , we have $\left\{q_{\iota} y_{\iota}\right\} \in S$. Since $0 \leqq q_{\iota} \leqq 1$, we have $q_{\iota}^{2} \leqq q_{\iota}$ and hence

$$
\sum\left|1-\left(p_{\imath} y_{\iota}, p_{\iota} q_{\iota} y_{\imath}\right)\right|=\sum\left|1-\left(y_{\iota}, q_{\imath} y_{\iota}\right)\right|
$$




$$
\begin{aligned}
& \leqq \Sigma\left|1-\left\|y_{\imath}\right\|^{2}\right|+\sum\left(y_{\iota},\left(1-q_{\imath}\right) y_{\iota}\right) \\
& \leqq \sum\left|1-\left\|y_{\imath}\right\|^{2}\right|+\sum\left(y_{\iota},\left(1-q_{\imath}^{2}\right) y_{\iota}\right) \\
& \leqq 2 \sum\left|1-\left\|y_{\iota}\right\|^{2}\right|+\Sigma\left|1-\left\|q_{\iota} y_{\iota}\right\|^{2}\right| \text {. }
\end{aligned}
$$

Since $\left\{y_{\imath}\right\} \in S$, we have $\sup \left\|y_{\iota}\right\|<\infty$ and hence

$$
\sum\left|1-\left\|q_{\imath} y_{\imath}\right\|^{2}\right| \leqq \sup \left(1+\left\|q_{\imath} y_{\imath}\right\|\right) \sum\left|1-\left\|q_{\imath} y_{\imath}\right\|\right|<\infty .
$$

Therefore $\left\{p_{\iota} y_{\iota}\right\} \sim\left\{p_{\iota} q_{\iota} y_{\iota}\right\}=\left\{v_{\iota} y_{\iota}\right\} \sim\left\{x_{\iota}\right\}$.

Let $s_{\iota}=s^{\prime}\left(y_{\iota}\right)-p_{\iota}^{*} p_{\iota}$. If $s_{\iota} y_{\iota}=0$, then we have $\left\{x_{\iota}\right\} \underset{p}{\sim}\left\{y_{\iota}\right\}$. In general, $s_{\iota}$ is a projection in $\Re_{\iota}^{\prime}$. Since $\left\{p_{\iota} y_{\iota}\right\} \in S$ by Lemma 1 , we have

$$
\begin{aligned}
& \sum\left\|s_{\iota} y_{\iota}\right\|^{2}=\sum\left(\left\|y_{\iota}\right\|^{2}-\left\|p_{\iota} y_{\iota}\right\|^{2}\right) \leqq \sum\left|1-\left\|y_{\iota}\right\|^{2}\right|+\sum\left|1-\left\|p_{\iota} y_{\iota}\right\|^{2}\right| \\
& \leqq \sup \left(1+\left\|y_{\imath}\right\|+\left\|p_{\imath} y_{\imath}\right\|\right) \sum\left(\left|1-\left\|y_{\imath}\right\|\right|+\left|1-\left\|p_{\imath} y_{\imath}\right\|\right|\right)<\infty \text {. }
\end{aligned}
$$

Hence $s_{\imath} y_{\iota}=0$, possibly except for a countable number of $\iota=\iota(l), l=1$, $2, \cdots$

Let $F_{\iota}$ be the central projection in $\Re_{\iota}^{\prime}$ such that $F_{\iota} p_{\imath}^{*} p_{\iota}$ is finite and $\left(1-F_{\iota}\right) p_{\iota}^{*} p_{\iota}$ is properly infinite in $\Re_{\iota}^{\prime}$. There exists a partial isometry $p_{\iota}^{\prime}$ in $\Re_{\iota}^{\prime}$ such that $p_{\iota}^{\prime *} p_{\iota}^{\prime}=F_{\iota}\left(1-p_{\iota}^{*} p_{\iota}\right), p_{\iota}^{\prime} p_{\iota}^{\prime *}=F_{\iota}\left(1-p_{\iota} p_{\iota}^{*}\right)$. There also exist projections $e_{\iota k}$ in $\Re_{\iota}^{\prime}, k=1,2, \ldots$ (countably infinite number) such that each $e_{\iota k}$ is equivalent to $\left(1-F_{\iota}\right) p_{\iota}^{*} p_{\iota}$ and $\sum_{k} e_{\iota k}=\left(1-F_{\iota}\right) p_{\iota}^{*} p_{\iota}$. Since $\sum_{k}\left\|e_{\imath k} y_{\imath}\right\|^{2} \leqq\left\|y_{\imath}\right\|^{2}$, there exists $k=k(l)$ such that $\left\|e_{\iota k} y_{\imath}\right\|^{2}<2^{-l}$ for $\iota=$ $\iota(l)$. Then there exist a partial isometry $p_{\iota(l)}^{\prime \prime}$ such that $p_{\iota(l)}^{\prime \prime *} p_{\iota(l)}^{\prime \prime}=e_{\iota(l) k(l)}$ $+\left(1-F_{\iota(l)}\right)\left(1-p_{\iota(l)}^{*} p_{\iota(l)}\right), p_{\iota(l)}^{\prime \prime} p_{\iota(l)}^{\prime \prime *}=\left(1-F_{\iota(l)}\right)\left(1-p_{\iota(l)}\left(1-e_{\iota(l) k(l)}\right) p_{\iota(l)}^{*}\right)$. Set $\bar{p}_{\iota}=p_{\iota}$ if $\iota \neq \iota(l), l=1,2, \cdots$, and $\bar{p}_{\iota}=F_{\iota}\left(p_{\iota}+p_{\iota}^{\prime}\right)+\left(1-F_{l}\right) p_{l}\left(1-e_{\iota k(l)}\right)$ $+p_{\iota}^{\prime \prime}$ for $\iota=\iota(l)$.

We first see from the construction that $\bar{p}_{\iota}$ is unitary for $\iota=\iota(l)$ and hence $\bar{p}_{\iota}^{*} \bar{p}_{\iota} y_{\iota}=y_{\iota}$ for all $\iota$. For $\iota \neq \iota(l), \bar{p}_{\iota} y_{\iota}=p_{\iota} y_{\iota}$. For $\iota=\iota(l)$, we have

$$
\begin{aligned}
& \left|\left(\bar{p}_{\iota} y_{\iota}, p_{\iota} y_{\iota}\right)-\left\|p_{\iota} y_{\iota}\right\|^{2}\right| \\
& \quad=\left|\left(\bar{p}_{\iota}\left(s_{\iota}+e_{\iota k(l)}\right) y_{\iota}, p_{\iota} e_{\iota k(l)} y_{\iota}\right)-\left\|p_{\iota} e_{\iota k(l)} y_{\iota}\right\|^{2}\right| \\
& \leqq\left(\left\|s_{\iota} y_{\iota}\right\|+\left\|e_{\iota k(l)} y_{\iota}\right\|\right)\left\|e_{\iota k(l)} y_{\iota}\right\|+\left\|e_{\iota k(l)} y_{\iota}\right\|^{2}
\end{aligned}
$$


which is summable over $l=1,2, \ldots$ Therefore

$$
\begin{aligned}
& \sum\left|1-\left(\bar{p}_{\imath} y_{\iota}, p_{\imath} y_{\imath}\right)\right| \\
& \quad \leqq \sum\left|1-\left\|p_{\iota} y_{\imath}\right\|^{2}\right|+\sum_{l}\left|\left(\bar{p}_{\iota(l)} y_{\iota(l)}, p_{\iota(l)} y_{\iota(l)}\right)-\left\|p_{\iota(l)} y_{\iota(l)}\right\|^{2}\right| \\
& \quad<\infty .
\end{aligned}
$$

Hence $\left\{\bar{p}_{\iota} y_{\iota}\right\} \in S$ by Lemma 1 and $\left\{\bar{p}_{\iota} y_{\iota}\right\} \sim\left\{p_{\iota} y_{\iota}\right\} \sim\left\{x_{\iota}\right\} . \quad$ Q.E.D.

Proof of Theorem (2). In the provious proof $\bar{p}_{\iota}$ is unitary for $c=$ $c(l)$. Hence this construction (even if $s_{\iota} y_{\iota}=0$ for all $\iota$ ) gives the equivalence of $\underset{u}{\sim}$ and $\underset{p}{\sim}$ when the index set $I$ is countable. Q.E.D.

Lemma 3. $\underset{p}{\sim}$ is an equivalence relation

Proof. Obviously $\left\{x_{\iota}\right\} \sim\left\{x_{\iota}\right\}$ because $\left\{x_{\iota}\right\} \sim\left\{p_{\iota} x_{\iota}\right\}$ with $p_{\iota}=1$. Suppose $\left\{x_{\imath}\right\} \sim\left\{p_{\iota} y_{\iota}\right\}$. Since $\left(y_{\iota}, p_{\imath}^{*} x_{\imath}\right)=\left(x_{\iota}, p_{\imath} y_{\iota}\right)^{*}$, we have $\left\{y_{\iota}\right\} \sim\left\{p_{\iota}^{*} x_{\iota}\right\}$ and hence $\left\{y_{\imath}\right\} \underset{v}{\sim}\left\{x_{\imath}\right\}$. By Lemma 2, $\left\{y_{\imath}\right\} \underset{p}{\sim}\left\{x_{\iota}\right\}$.

Finally, suppose $\left\{x_{\iota}\right\} \sim\left\{p_{\iota} y_{\iota}\right\}$ and $\left\{y_{\iota}\right\} \sim\left\{p_{\iota}^{\prime} z_{\iota}\right\}$ with $p_{\iota}^{*} p_{\iota} y_{\iota}=y_{\iota}$. Then $\left(p_{\iota} y_{\iota}, p_{\iota} p_{\iota}^{\prime} z_{\iota}\right)=\left(p_{\imath}^{*} p_{\iota} y_{\iota}, p_{\iota}^{\prime} z_{\iota}\right)=\left(y_{\iota}, p_{\iota}^{\prime} z_{\iota}\right)$. Hence $\left\{x_{\iota}\right\} \sim\left\{p_{\iota} y_{\iota}\right\} \sim$ $\left\{p_{\iota} p_{\iota}^{\prime} z_{\iota}\right\}$. Therefore $\left\{x_{\iota}\right\} \underset{v}{\sim}\left\{z_{\iota}\right\}$ and by Lemma $2,\left\{x_{\iota}\right\} \underset{p}{\sim}\left\{z_{\iota}\right\}$.

Q.E.D.

\section{§3. Central Support $E(c)$}

Lemma 4. For $\mathfrak{c}, \mathfrak{c}^{\prime} \in \mathfrak{S}_{0}$, either $E(\mathfrak{c}) E_{\mathrm{c}^{\prime}}=E_{\mathfrak{c}^{\prime}}$ or $E(\mathfrak{c}) E_{\mathrm{c}^{\prime}}=0$.

Proof. Take any $\left\{y_{\imath}\right\} \in S_{0}$. By Lemma 4.2 of $[6]$,

$$
E(\mathfrak{c})\left(\otimes y_{\imath}\right)=\lim _{J \subset \subset I} E_{J}\left(\otimes y_{\imath}\right)
$$

where $J \subset \subset I$ indicates that $J$ is a finite subset of $I$ and $E_{J}$ is the smallest projection in

$$
\Re\left(J^{c}\right)=\left(\bigcup_{\iota \notin J} \pi\left(\Re_{\iota}\right)\right)^{\prime \prime}
$$


scuh that $E_{J}\left(\otimes x_{\imath}\right)=\otimes x_{\imath}$ for a fixed $\left\{x_{\imath}\right\} \in c$. Let $c^{\prime}=\mathfrak{c}\left(\left\{y_{\imath}\right\}\right)$. Since $H_{\mathrm{c}^{\prime}}$ is invariant under $\Re \supset \Re\left(J^{c}\right)$, each $E_{J}\left(\otimes y_{\imath}\right)$ as well as its limit $E(\mathfrak{c})$ $\left(\otimes y_{\imath}\right)$ is in $H_{c^{\prime}}$.

By Lemma 3.1 of $[2]$, there exists $J$ for any given $\varepsilon>0$ such that $J \subset \subset I$ and

$$
\left\|E(\mathrm{c})\left(\otimes y_{\imath}\right)-z_{J} \otimes y\left(J^{c}\right)\right\|<\varepsilon
$$

where $z_{J} \in \bigotimes_{\iota \in J} H_{\iota}$ and $y\left(J^{c}\right)=\bigotimes_{\iota \notin J} y_{\imath}$. For the same $\varepsilon$ and $J$, there exists $K \supset J, K \subset \subset I$ such that

$$
\left\|E(c)\left(\otimes y_{\iota}\right)-E_{K}\left(\otimes y_{\imath}\right)\right\|<\varepsilon
$$

Since $E_{K} \in \Re\left(K^{c}\right) \subset \Re\left(J^{c}\right)$, we can write $E_{K}\left(\otimes y_{\iota}\right)=y(J) \otimes z$ for some $z \in \bigotimes_{\iota \notin J} H_{\iota}$. From the two inequalities,

$$
\left\|z_{J} \otimes y\left(J^{c}\right)-y(J) \otimes z\right\|<2 \varepsilon
$$

Since $\left\{y_{\imath}\right\} \in S_{0}, a_{2}=\left\|y\left(J^{c}\right)\right\|$ and $b_{1}=\|y(J)\|$ are bounded away from 0 and $\infty$ when $J$ runs over all finite subsets of $I$. Let $a=\left\|E(\mathfrak{c})\left(\otimes y_{\imath}\right)\right\|$ and assume that $a \neq 0$. Then we have from (3.2) and (3.3), $\left|a_{1}-a / a_{2}\right|$ $<\varepsilon / a_{2}$ and $\left|b_{2}-a / b_{1}\right|<\varepsilon / b_{1}$ for $a_{1}=\left\|z_{J}\right\|$ and $b_{2}=\|z\|$. Therefore $a_{1}$ and $b_{2}$ are also bounded away from 0 and $\infty$ for sufficiently small $\varepsilon$. From (3.4), we also have $\left|a_{1} a_{2}-b_{1} b_{2}\right|<2 \varepsilon$.

We set $\Phi_{1}=z_{J} / a_{1}, \Phi_{2}=y\left(J^{c}\right) / a_{2}, \Psi_{1}=y(J) / b_{1}, \Psi_{2}=z / b_{2} . \quad$ They are all unit vectors. From (3.4), we obtain, by using $\left|a_{1} a_{2}-b_{1} b_{2}\right|<2 \varepsilon$ and separation of $a_{1} a_{2}, b_{1} b_{2}$ from 0 ,

$$
\left\|\Phi_{1} \otimes \Phi_{2}-\Psi_{1} \otimes \Psi_{2}\right\|<\varepsilon^{\prime}(\varepsilon)
$$

where $\varepsilon^{\prime}(\varepsilon) \rightarrow 0$ as $\varepsilon \rightarrow 0$. Then

$$
1-\operatorname{Re}\left(\Phi_{1}, \Psi_{1}\right)\left(\Phi_{2}, \Psi_{2}\right)<\varepsilon^{\prime}(\varepsilon)^{2} / 2
$$

Since $\left|\left(\Phi_{1}, \Psi_{1}\right)\right| \leqq 1$ and $\left|\left(\Phi_{2}, \Psi_{2}\right)\right| \leqq 1$, we have

$$
\begin{aligned}
& \varepsilon^{\prime}(\varepsilon)^{2} / 2>1-\left|\left(\Phi_{1}, \Psi_{1}\right)\right|\left|\left(\Phi_{2}, \Psi_{2}\right)\right| \\
& \geqq \max \left(1-\left|\left(\Phi_{1}, \Psi_{1}\right)\right|, 1-\left|\left(\Phi_{2}, \Psi_{2}\right)\right|\right) .
\end{aligned}
$$


Hence choosing $\theta$ and $\theta^{\prime}$ such that $\left(\Phi_{1}, e^{i \theta} \Psi_{1}\right)$ and $\left(\Phi_{2}, e^{i \theta^{\prime}} \Psi_{2}\right)$ are both non-negative, we have

$$
\left\|\Phi_{1}-e^{i \theta} \Psi_{1}\right\|_{1}<\varepsilon^{\prime}(\varepsilon),\left\|\Phi_{2}-e^{i \theta^{\prime}} \Psi_{2}\right\|<\varepsilon^{\prime}(\varepsilon) .
$$

In particular, we use the first inequality and (3.2) to obtain

$$
\left\|E(\mathrm{c})\left(\otimes y_{\iota}\right)-\lambda_{\varepsilon}\left(\otimes y_{\imath}\right)\right\|<\varepsilon+a_{1} a_{2} \varepsilon^{\prime}(\varepsilon)
$$

where $\lambda_{\varepsilon}=e^{i \theta} a_{1} / b_{1}$ is a complex number depending on $\varepsilon$. We choose a sequence $\varepsilon_{n} \rightarrow 0$ such that $\lambda_{\varepsilon_{n}} \rightarrow \lambda$, which is possible because $\lambda_{\varepsilon}$ is bounded. Then, by using separation of $a_{1} a_{2}$ from $\infty$,

$$
E(\mathfrak{c})\left(\otimes y_{\imath}\right)=\lambda\left(\otimes y_{\imath}\right)
$$

In this derivation, we assumed $\left\|E(\mathfrak{c})\left(\otimes y_{\imath}\right)\right\| \neq 0$. If this is not the case (3.5) holds with $\lambda=0$. Since $E(c))^{2}=E(c)$, we have $\lambda^{2}=\lambda$ and hence $\lambda=1$ or 0 .

If $c\left(\left\{y_{\imath}\right\}\right)=c\left(\left\{y_{\imath}^{\prime}\right\}\right)$, then by Lemma 3.1 of $[2]$, there exists $J \subset \subset I$ such that

$$
\left\|\otimes y_{\imath}^{\prime}-z^{\prime} \otimes y\left(J^{c}\right)\right\|<\varepsilon
$$

By $(3.1), E(c)\left(\otimes y_{\imath}\right)=\lambda\left(\otimes y_{\imath}\right)$ with $\lambda=1$ or 0 implies

$$
\lim _{K} E_{K}\left(z^{\prime} \otimes y\left(J^{c}\right)\right)=\lambda\left(z^{\prime} \otimes y\left(J^{c}\right)\right)
$$

and hence by (3.1) and (3.6), we have $E(c)\left(\otimes y_{\imath}^{\prime}\right)=\lambda\left(\otimes y_{\imath}^{\prime}\right)$ with the same $\lambda$. Hence $E(\mathrm{c}) E_{\mathrm{c}^{\prime}}=\lambda E_{\iota^{\prime}}$ with $\lambda=1$ or 0 .

Q.E.D.

Let $\pi_{c}$ denote the restriction of the representation $\pi$ to $E_{\mathrm{c}} H$.

Lemma 5. Let $\mathfrak{c}, \mathfrak{c}^{\prime} \in S_{0} . \quad$ Either $E(\mathfrak{c})=E\left(\mathfrak{c}^{\prime}\right)$ or $E(\mathfrak{c}) \perp E\left(\mathfrak{c}^{\prime}\right) . \quad A c$ cordingly, $\pi_{\mathrm{c}}$ and $\pi_{\mathrm{c}^{\prime}}$ are either quasi-equivalent or disjoint.

Proof. The first part follows from Lemma 4. It then implies the second part.

Q.E.D.

Proof of Theorem (1). First assume that $\underset{\mathfrak{c}^{\prime}}{\sim} \underset{p}{\mathcal{c} .}$ Let $x_{\iota}$ and $y_{\iota}$ in 
$H_{\iota}$ be such that $\mathrm{c}=\mathfrak{c}\left(\left\{x_{\iota}\right\}\right), \mathrm{c}^{\prime}=\mathfrak{c}\left(\left\{y_{\iota}\right\}\right),\left\|x_{\iota}\right\|=\left\|y_{\imath}\right\|=1$, and $\left\{x_{\iota}\right\} \sim\left\{p_{\iota} y_{\iota}\right\}$ for partial isometries $p_{\iota}$ with $p_{\iota}^{*} p_{\iota} y_{\iota}=y_{\iota}$. Let $\omega_{z}$ generally denote the vector state by $z$. Then $\omega_{\otimes x_{\imath}}=\otimes \omega_{x_{\imath}}$ and $\omega_{\otimes y_{\imath}}=\otimes \omega_{y_{i}}$.

Let $\otimes p_{\imath}$ be the mapping from $H_{\mathfrak{c}^{\prime}}$ to $H_{\mathrm{c}}$ defined by

$$
\left(\otimes p_{\iota}\right)\left(y\left(J^{c}\right) \otimes z\right)=(p y)\left(J^{c}\right) \otimes p(J) z
$$

where $J$ is any finite index set, $y\left(J^{c}\right)=\bigotimes_{\iota \notin J} y_{\iota},(p y)\left(J^{c}\right)=\bigotimes_{\iota \notin J} p_{\iota} y_{\iota}, p(J)=$ $\bigotimes_{\iota \in J} p_{\iota}$ and $z \in \bigotimes_{\iota \in J} H_{\iota}$. If $\left\{p_{\iota} y_{\iota}\right\} \sim\left\{x_{\iota}\right\}$, then $p=\otimes p_{\iota}$ satisfies $p H_{\iota^{\prime}} \subset H_{\iota}$ and $\|p\| \leqq 1, \pi_{c}(Q) p=p \pi_{c^{\prime}}(Q)$ for $Q \in \Re$ and hence for $Q \in \Re$. Furthermore $p\left(\otimes y_{\imath}\right)=\otimes p_{\iota} y_{\iota} \neq 0$. Hence $\pi_{c}$ and $\pi_{c^{\prime}}$ have a nonzero intertwining operator $p$ and hence are not disjoint. By Lemma 5 , we have $E(\mathfrak{c}) E_{\mathrm{c}^{\prime}}=$ $E_{c^{\prime}}$.

Conversely, assume $E(c) E_{c^{\prime}}=E_{c^{\prime}}$. Then $\pi_{c}$ and $\pi_{c^{\prime}}$ are quasi-equivalent by Lemma 5 . If $x_{\iota}$ satisfies $\left\|x_{\iota}\right\|=1, \mathfrak{c}\left(\left\{x_{\iota}\right\}\right)=c$, then there exist a countable number of vectors $\xi_{l}$ in $H_{c^{\prime}}$ such that $\omega_{\otimes x_{l}}=\sum_{l} \omega_{\zeta_{l}}$. Since product vectors are total in $H_{\mathfrak{c}^{\prime}}$, there exists $y_{\iota} \in H_{\iota}$ such that $\left\|y_{\imath}\right\|=1$, $\mathfrak{c}\left(\left\{y_{\imath}\right\}\right)=\mathfrak{c}^{\prime}$ and $\left(\xi_{1}, \otimes y_{\imath}\right) \neq 0$. Then

$$
\left\|\omega_{\otimes x_{\iota}}-\omega_{\otimes y_{\imath}}\right\| \leqq \sum_{l \neq 1}\left\|\xi_{l}\right\|^{2}+\left\|\omega_{\xi_{1}}-\omega_{\otimes y_{l}}\right\|<2
$$

Let $\left\|\omega_{\otimes x_{c}}-\omega_{\otimes y_{t}}\right\|_{J}$ denote the norm of the restricition of $\omega_{\otimes x_{c}}-\omega_{\otimes y_{c}}$ to $\Re(J)=\left(\bigcup_{\iota \in J}\left(\Re_{\iota}\right)\right)^{\prime \prime}$. By proposition 1.12 and Corollary 2.6 of [5], we have

$$
\begin{aligned}
\prod_{\iota \in J} \rho\left(\omega_{x_{\iota}}, \omega_{y_{\iota}}\right) & \geqq 2^{-1}\left(2-\left\|\omega_{\otimes x_{\iota}}-\omega_{\otimes y_{\iota}}\right\|_{J}\right) \\
& \geqq 2^{-1}\left(2-\left\|\omega_{\otimes x_{\iota}}-\omega_{\otimes y_{\iota}}\right\|\right)>0
\end{aligned}
$$

where $\rho(\mu, \nu)=2^{-1}\left(\mu(1)+\nu(1)-d(\mu, \nu)^{2}\right)=2^{-1}\left(2-d(\mu, \nu)^{2}\right)$ for states $\mu$ and $\nu$. Since each $\rho\left(\omega_{x_{t}}, \omega_{y_{\imath}}\right)$ is in the interval $[0,1]$, (3.9) for arbitrary $J$ implies the absolute convergence of $\Pi \rho\left(\omega_{x_{t}}, \omega_{y_{\imath}}\right)$ and hence

$$
\sum d\left(\omega_{x_{\iota}}, \omega_{y_{\iota}}\right)^{2}=2 \sum\left(1-\rho\left(\omega_{x_{\iota}}, \omega_{y_{\iota}}\right)\right)<\infty
$$

By Theorem 4 of $[1]$, there exist $x_{\iota}^{\prime}$ and $y_{\imath}^{\prime}$ in $H_{\iota}$ such that 


$$
\begin{gathered}
\omega_{x_{\imath}^{\prime}}=\omega_{x_{\imath}}, \omega_{y_{\imath}^{\prime}}=\omega_{y_{\imath}}, \quad\left\|x_{\imath}^{\prime}-y_{\imath}^{\prime}\right\|=d\left(\omega_{x_{\iota}}, \omega_{y_{\imath}}\right), \\
\left(x_{\iota}^{\prime}, y_{\imath}^{\prime}\right)>0 .
\end{gathered}
$$

Since $\omega_{x_{\imath}^{\prime}}=\omega_{x_{\iota}}, p_{\iota} s^{\prime}\left(x_{\imath}\right)=p_{\iota}$ and $p_{\iota} Q x_{\iota}=Q x_{\iota}^{\prime}$ for all $Q \in \Re_{\iota}$ defines (by continuity) a partial isometry $p_{\iota} \in \mathfrak{R}_{\iota}^{\prime}$, which satisfies $p_{\iota}^{*} p_{\iota} x_{\iota}=x_{\iota}, x_{\iota}^{\prime}=p_{\iota} x_{\iota}$. Similarly there exists a partial isometry $p_{\iota}^{\prime} \in \Re_{\iota}^{\prime}$ such that $p_{\iota}^{\prime *} p_{\iota}^{\prime} y_{\iota}=y_{\iota}$ and $y_{\iota}^{\prime}=p_{\iota}^{\prime} y_{\iota}$. From (3.10) and (3.11),

$$
\sum\left|1-\left(x_{\iota}^{\prime}, y_{\imath}^{\prime}\right)\right|=2^{-1} \sum\left\|x_{\iota}^{\prime}-y_{\imath}^{\prime}\right\|^{2}<\infty
$$

and hence $\left\{x_{\imath}\right\} \underset{p}{\sim}\left\{p_{\iota} x_{\iota}\right\} \sim\left\{p_{\iota}^{\prime} y_{\iota}\right\} \underset{p}{\sim}\left\{y_{\iota}\right\}$. Therefore $\left\{x_{\imath}\right\} \underset{p}{\sim}\left\{y_{\iota}\right\}$ by Lemma 3.

Q.E.D.

\section{§4. Discussions}

If $\left\{x_{\iota}\right\} \underset{p}{\sim}\left\{y_{\iota}\right\}$, then $x_{\iota}=p_{\iota} y_{\iota}$ for a partially isometric $p_{\iota} \in \Re_{\iota}^{\prime}$ for all c except for a countable number of $\iota$, where $p_{\iota}$ satisfies $p_{\iota}^{*} p_{\iota} y_{\iota}=y_{\iota}$. (Note that $\left\|x_{\imath}\right\|=\left\|y_{\imath}\right\|=1, \quad\left(x_{\iota}, y_{\imath}\right)=1$ imply $\left\|x_{\iota}-y_{\imath}\right\|_{1}^{2}=0$ and hence $x_{\iota}=y_{\imath}$. Then $s^{\prime}\left(x_{\iota}\right)$ and $s^{\prime}\left(y_{\iota}\right)$ are equivalent in $\Re_{\iota}^{\prime}$. $p_{\iota}$ can be extended to a unitary in $\Re_{\iota}^{\prime}$ if and only if $1-s^{\prime}\left(x_{\iota}\right)$ and $1-s^{\prime}\left(y_{\iota}\right)$ are equivalent in $\Re_{\iota}^{\prime}$.

If $\left\{x_{\iota}\right\} \underset{u}{\sim}\left\{y_{\iota}\right\}$, then $x_{\iota}=u_{\iota} y_{\iota}$ for a unitary $u_{\iota} \in \Re_{\iota}^{\prime}$ for all $\iota$ except for a countable number of $c$. Therefore both $s^{\prime}\left(x_{\iota}\right)$ and $1-s^{\prime}\left(x_{\iota}\right)$ are equivalent to $s^{\prime}\left(y_{\imath}\right)$ and $1-s^{\prime}\left(y_{\imath}\right)$ respectively, with a countable exception.

Due to Theorem (2) and its proof, the above argument gives the following:

Theorem (3). $\quad\left\{x_{\iota}\right\} \underset{u}{\sim}\left\{y_{\iota}\right\}$ if and only if $\left\{x_{\iota}\right\} \underset{p}{\sim}\left\{y_{\iota}\right\}$ and $1-s^{\prime}\left(x_{\iota}\right)$ is equivalent to $1-s^{\prime}\left(y_{\iota}\right)$ in $\Re_{\iota}^{\prime}$ for all c except for a countable many $\iota$, where $s^{\prime}\left(x_{\iota}\right)$ is the support projection of $x_{\iota}$ in $\Re_{\iota}^{\prime} . \quad c\left(\left\{x_{\iota}\right\}\right) \underset{u}{\sim} \mathfrak{c}\left(\left\{y_{\iota}\right\}\right)$ if and only if $\left\{x_{\iota}\right\} \underset{u}{\sim}\left\{y_{\iota}\right\}$.

Proof. The first half is already shown. By definition, if $\left\{x_{\iota}\right\} \sim_{u}\left\{y_{\iota}\right\}$, then $\mathfrak{c}\left(\left\{x_{\iota}\right\}\right) \underset{u}{\sim} \mathfrak{c}\left(\left\{y_{\imath}\right\}\right)$. Therefore it remains to show that $\mathfrak{c}\left(\left\{x_{\iota}\right\}\right) \underset{u}{\sim} \mathfrak{c}\left(\left\{y_{\imath}\right\}\right)$ 
implies $\left\{x_{\iota}\right\} \underset{u}{\sim}\left\{y_{\imath}\right\}$, which is rather trivial consequence of Definition:

By definition, $\mathfrak{c}\left(\left\{x_{\imath}\right\}\right) \underset{u}{\mathcal{c}}\left(\left\{y_{\imath}\right\}\right)$ implies the existence of $x_{\iota}^{\prime}$ and $y_{\imath}^{\prime} \in$

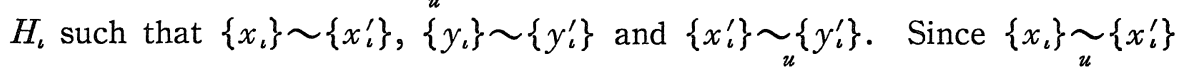
and $\left\{y_{\iota}\right\} \underset{u}{\sim}\left\{y_{\iota}^{\prime}\right\}$ trivially, it follows that $\left\{x_{\iota}\right\} \underset{u}{\sim}\left\{y_{\iota}\right\} . \quad$ Q.E.D.

Example. Suppose $x_{\iota}$ are cyclic for $\Re_{\iota}$ and $p_{\iota}$ are isometric operators in $\Re_{\iota}^{\prime}$, which are not unitaries. (This can happen for non-finite $\Re_{\iota}^{\prime}$.) Then $1-s^{\prime}\left(x_{\iota}\right)=0$ because $s^{\prime}\left(x_{\iota}\right) H$ is the closure of $\Re_{\iota} x_{\iota}$ and $x_{\iota}$ is cyclic. For $y_{\iota}=p_{\iota} x_{\iota}, 1-s^{\prime}\left(y_{\iota}\right)=1-p_{\iota} p_{\iota}^{*} \neq 0$. Hence, if the index set is noncountable, then $\left\{x_{\iota}\right\} \sim_{p}\left\{y_{\iota}\right\}$ but $\left\{x_{\iota}\right\}$ is not $u$-equivalent to $\left\{y_{\iota}\right\}$.

In this example, the representation of $\Re$ in $H_{\mathfrak{c}}$ and $H_{\mathfrak{c}^{\prime}}, \mathfrak{c}=\mathfrak{c}\left(\left\{x_{\imath}\right\}\right)$, $\mathfrak{c}^{\prime}=\mathfrak{c}\left(\left\{y_{\imath}\right\}\right)$, are not unitarily equivalent as is seen by the following argument:

Let $y_{\iota \lambda}, \lambda \in \Lambda_{\iota}$ be an orthonormal basis for $H_{\iota}$ such that $y_{\iota 0}=y_{\iota}$. Then $\otimes y_{\iota \kappa(\iota)}$, with $\kappa(\iota)=0$ except for a finite number of $\iota$, is an orthonormal basis for $H_{\mathfrak{c}^{\prime}}$. Any $z \in H_{\mathfrak{c}^{\prime}}$ has only a countable number of nonzero components on this basis and hence $z=\left(\bigotimes_{\iota \notin A} y_{\iota}\right) \otimes z^{\prime}, z^{\prime} \in \bigotimes_{\iota \in A} H_{\iota}$ for some countable index set $A$. Since $R=\left(\bigcup R_{\iota}\right)^{\prime \prime}$ and $y_{\iota}$ is not cyclic for $\Re_{\iota}, z$ can not be cyclic for $\Re$ in $H_{\mathfrak{c}^{\prime}}$. On the other hand, $\otimes x_{\iota} \in H_{c}$ is cyclic for $\Re$ in $H_{\mathfrak{c}}$. Hence $\Re \mid H_{\mathfrak{c}}$ and $\Re \mid H_{\mathfrak{c}^{\prime}}$ can not be unitarily equivalent.

Theorem (4). $\pi_{\mathfrak{c}}$ is quasi-equivalent to $\pi_{\mathfrak{c}^{\prime}}$ if and only if $\underset{p}{\mathfrak{c} \sim \mathfrak{c}^{\prime} .} \pi_{\mathrm{c}}$ is unitarily equivalent to $\pi_{\mathfrak{c}^{\prime}}$ if $\mathfrak{c}_{u}^{\mathcal{c}} \%$ If the index set is countable, then $\pi_{\mathrm{c}}$ is unitarily equivalent to $\pi_{\mathfrak{c}^{\prime}}$ if and only if $\pi_{\mathrm{c}}$ is quasi-equivalent to $\pi_{\mathrm{c}^{\prime}}$.

Proof. The first part is obvious by Lemma 5 and Theorem (1). To see the second part, assume $\mathfrak{c}=\mathfrak{c}\left(\left\{x_{\iota}\right\}\right), \mathfrak{c}^{\prime}=\mathfrak{c}\left(\left\{y_{\iota}\right\}\right)$ and $\left\{x_{\iota}\right\} \sim\left\{u_{\iota} y_{\iota}\right\}$, where $u_{\iota} \in \Re_{\imath}^{\prime}$ is unitary. Then $\otimes u_{\iota}$ defined by the same equation as $\otimes p_{\iota}$ in the proof of Lemma 5 is obviously isometric and its range contains all $(u y)\left(J^{c}\right) \otimes u(J) H(J)$ where $H(J)=\bigotimes_{\iota \in J} H_{\iota}$. Since $u(J)$ is unitary $u(J) H$ $(J)=H(J)$ and since $\otimes\left(u_{\imath} y_{\imath}\right) \in H_{\mathfrak{c}}$, the image of $\otimes u_{\iota}$ is the whole $H_{\imath}$. 
Hence $\otimes u_{\iota}$ is a unitary intertwining operator for $\pi_{c}$ and $\pi_{c^{\prime}}$, which proves the unitary equivalence of $\pi_{c}$ and $\pi_{c^{\prime}}$. The last part follows then from Theorem (2).

Q.E.D.

Remark. The unitary equivalence of $\pi_{c}$ and $\pi_{c^{\prime}}$ does not necessarily imply $\underset{u}{\mathfrak{c}} \mathfrak{c}^{\prime}$. For example, consider $\mathfrak{R}_{r}=\mathscr{B}\left(H_{r}^{\prime}\right) \otimes \mathbb{1}^{\prime \prime}$ on $H_{r}^{\prime} \otimes H_{r}^{\prime \prime}=H_{r}$ with all real $r \neq 0$ as index set and $x_{r}=\sum_{k=1}^{\infty} 2^{-k} e_{k} \otimes e_{k}, y_{r}=\sum_{k=1}^{\infty} 2^{-k} e_{k} \otimes e_{k+1}$ for $r>0$ and $x_{r}=\sum_{k=1}^{\infty} 2^{-k} e_{k} \otimes e_{k+1}, y_{r}=\sum_{k=1}^{\infty} 2^{-k} e_{k} \otimes e_{k}$ for $r<0$, where all $H_{r}^{\prime}$ and $H_{r}^{\prime \prime}$ are identified with a single Hilbert space $H$ and $\left\{e_{k}\right\}$ is its orthonormal basis. Then obviously $\left\{x_{\iota}\right\} \underset{u}{\sim}\left\{y_{\iota}\right\}$ does not hold but $\pi_{c}$ is unitarily equivalent to $\pi_{c^{\prime}}$ for $\mathfrak{c}=\mathfrak{c}\left(\left\{x_{\iota}\right\}\right)$ and $\mathfrak{c}^{\prime}=\mathfrak{c}\left(\left\{y_{\iota}\right\}\right)$.

For $Q_{\iota} \in \mathscr{B}\left(H_{\imath}\right)$ with $\Pi\left\|Q_{\imath}\right\|<+\infty$, there exists a unique bounded linear operator $\otimes Q_{\imath}$ on $\otimes H_{\imath}=H$ satisfying $\left(\otimes Q_{\imath}\right)\left(\otimes x_{\imath}\right)=\otimes Q_{\imath} x_{\imath}$ for all $\left\{x_{\imath}\right\} \in S_{0}$ by Theorem 3.1 in $[6]$, where $\otimes Q_{\imath} x_{\iota}=0$ if $\left\{Q_{\imath} x_{\iota}\right\} \notin S_{0}$. If $Q_{\imath}^{\prime} \in \Re_{\imath}^{\prime}$ and $\Pi\left\|Q_{\imath}^{\prime}\right\|<+\infty, \otimes Q_{\imath}^{\prime}$ can be defined in exactly the same manner and $\otimes Q_{\iota}^{\prime} \in \Re^{\prime}$ by Theorem 3.2 in [6].

$\otimes p_{\iota}$ in the proof of Theorem (1) is this $\otimes p_{\iota}$ with its domain restricted to $H_{c}^{\prime}$.

Theorem (5). $\Re^{\prime}$ is generated by the set of all $E_{\mathrm{c}}, \mathrm{c} \in \mathfrak{S}_{0}$ and $\otimes p_{\mathrm{c}}$ with partial isometries $p_{\iota} \in \Re_{\iota}^{\prime}$. If the index set is countable, $p_{\iota}$ can be restricted to unitaries.

Proof. Let $\mathfrak{N}$ be the set of all $E_{c}, c \in \mathfrak{S}_{0}$ and $\otimes p_{\iota}$ with partial isometries $p_{\iota} \in \Re_{\iota}^{\prime}$. Since $\mathfrak{N} \subset \mathfrak{R}^{\prime}$, it is enough to prove that $Q \in \mathfrak{N}^{\prime}$ implies $Q \in \Re$. Let $Q \in \mathfrak{N}^{\prime}$.

Since isometries $p_{\iota} \in \Re_{\iota}^{\prime}$ generates $\Re_{\iota}^{\prime}$,

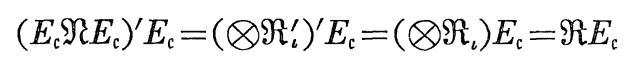

by Lemma 6.10 of $[3]$. Since $Q E_{\mathrm{c}}$ belongs to this set, there exists $Q_{1} \in \Re$ such that $Q E_{\mathrm{c}}=Q_{1} E_{\mathrm{c}}$. Let $\otimes y_{\imath} \in E(\mathrm{c}) H . \quad$ By Theorem (1) and Lemma 3, there exist partial isometries $p_{\iota} \in \Re_{\iota}^{\prime}$ such that $c\left(\left\{p_{\iota} y_{\iota}\right\}\right)=c$ and $p_{\imath}^{*} p_{\iota} y_{\iota}=$ $y_{\imath}$. Then $Q \otimes p_{\iota} y_{\iota}=Q_{1} \otimes p_{\imath} y_{\iota}$. Hence 


$$
\begin{aligned}
& Q \otimes y_{\iota}=Q\left(\otimes p_{\imath}^{*}\right) \otimes p_{\imath} y_{\iota}=\left(\otimes p_{\imath}^{*}\right) Q \otimes p_{\imath} y_{\iota} \\
& =\left(\otimes p_{\imath}^{*}\right) Q_{1} \otimes p_{\imath} y_{\iota}=Q_{1}\left(\otimes p_{\imath}^{*}\right) \otimes p_{\imath} y_{\iota}=Q_{1} \otimes y_{\iota} .
\end{aligned}
$$

This shows that $Q E(\mathfrak{c})=Q_{1} E(\mathrm{c}) \in \Re$. Hence $Q=\sum Q E(\mathrm{c}) \in \Re$ where the sum is over distinct $E(\mathrm{c})$.

If the index set is countable, then $p_{c}$ in the above argument can be taken to be unitaries by Theorem (2) and the latter half of Theorem (5) is obtained.

Q.E.D.

\section{Acknowledgement}

This work was partially inspired by the talk of Professor D. Bures at First Canadian Annual Symposium on Operator Algebras and their Applications at University of Tronto on March 31 - April 2, 1972. One of the authors (H. A) would like to thank Professor I. Halperin for his kind invitation to the Symposium and Professor Bures for discussion. One of the authors (H. A) would also like to thank Professor A. J. Coleman and Professor E. J. Woods for their warm hospitality at Department of Mathematics, Queen's University.

\section{References}

[1] Araki, H., Bures distance and a generalization of Sakai's non commutative Radon-Nikodym theorem.

[2] Araki, H. and E.J. Woods, Publ. RIMS, Kyoto Univ. 2 (1966), 157-242.

[3] Araki, H. and E.J. Woods, Publ. RIMS, Kyoto Univ. 4 (1968), 51-130.

[4] Bures, D., Pacific J. Math. 27 (1968), 13-37.

[5] Bures, D., Trans. Amer. Math. Soc. 135 (1969), 199-212.

[6] Nakagami, Y., Kodai Math. Sem. Rep. 22 (1970), 341-354.

[7] Nakagami, Y., Publ. RIMS, Kyoto Univ. 6 (1970), 257-292.

[8] von Neumann, J., Compositio Math. 6 (1937), 1-77. 